\title{
Nephrectomy - A study of recent trends in a high-volume tertiary care center, exploring the need for screening and preventive strategies
}

\author{
Ankit Vyas ${ }^{1}$, Kamlesh Singh ${ }^{2}$, Tarun Rochlani ${ }^{3}$, Sachin Bhujbal ${ }^{4}$, Bhushan Patil ${ }^{5}$, \\ Sujata Patwardhan ${ }^{6}$ \\ ${ }^{1,2,3}$ Mch Urology Resident, ${ }^{4}$ Assistant Professor, ${ }^{5}$ Additional Professor, ${ }^{6}$ Professor, Department of Urology, Seth GS \\ Medical College and KEM Hospital, Mumbai, Maharashtra, India
}

Background: Nephrectomy being one of the most commonly performed procedures, a retrospective study and analysis was done to evaluate, the scope for screening and timely preventive measures that could help to reduce the need for nephrectomy. Aims and Objectives: The aim of the study was to study indications, demographic-details, clinicalpresentations, time delay, comorbidities, operative procedures, and complications of patients undergoing nephrectomies. The study was to identify the high risk groups, assess for feasibility for screening measures, and formulate strategies for nephrectomy prevention for benign etiologies. Materials and Methods: A retrospective study was done on medical records of 220 consecutive nephrectomies from June 2018 to June 2020. Results: Out of 220 nephrectomies, $68 \%$ were performed for benign conditions, $17 \%$ for malignant etiology, and $15 \%$ were donor nephrectomies. In the benign group, the most common etiology was renal stones (44.54\%), followed by pelvic ureteric junction obstruction (12.72 \%), and renal tuberculosis $(6.81 \%)$. The most common clinical presentations were flank pain $(80 \%)$, followed by lower urinary tract symptoms (22\%), dysuria $(25 \%)$, hematuria $(15 \%)$, and fever (7\%). In the malignant group, the most common etiology was renal cell carcinoma $(14.09 \%)$. The most common clinical presentations were flank pain $(90 \%)$ followed by hematuria $(67 \%)$. Overall, $24 \%$ of the patients presented with acute kidney injury. A pre-operative intervention such as DJ stenting and percutaneous nephrostomy insertion was performed in $13 \%$ and $9 \%$ of patients, respectively. A laparoscopic approach was used in $76 \%$ of the patients out of which $10 \%$ were converted to open procedures. Partial nephrectomy was performed in $3 \%$ of patients. Conclusion: There is a difference in nephrectomy indications between Western countries and India, where $68 \%$ of nephrectomies are performed for benign conditions especially calculus disease. Nephrectomy for calculus disease is potentially preventable and public education in the form of posters, media and the involvement of the community medicine department could be helpful.

Key words: Benign; Calculus disease; Nephrectomy; Preventive strategies; Screening

\section{Access this article online}

\section{Website:}

http://nepjol.info/index.php/AJMS

DOI: 10.3126/ajms.v13i2.40434

E-ISSN: 2091-0576

P-ISSN: $2467-9100$

Copyright (c) 2022 Asian Journal of Medical Sciences

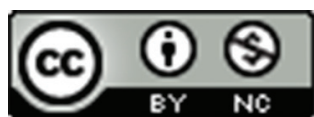

This work is licensed under a Creative Commons Attribution-NonCommercial 4.0 International License.

\section{INTRODUCTION}

Nephrectomy is one of the most commonly performed surgeries in the field of urology. However, its incidence, indications, presentations, and complications vary considerably among different geographical areas. There is a paucity of likewise data from the Indian subcontinent region. Here, we present an observational study of all nephrectomy surgeries performed at a high-volume tertiary care institute in a metropolitan city in the past 2 years. Our objective was to gain information about the demographic profile, complications, and common denominator in the series for self-learning. We also evaluated whether a certain group of the patients could be treated better, the scope for screening, and the need for any awareness campaigns. Emphasis was placed on what can be done to prevent 
these nephrectomies with a special focus on patients with calculus disease.

\section{Aims and objectives}

To study indications, demographic-details, clinicalpresentations, time delay, co-morbidities, operative procedures, and complications of patients undergoing nephrectomies. To identify high risk groups, assess for feasibility for screening measures and formulate strategies for nephrectomy prevention for benign etiologies.

\section{MATERIALS AND METHODS}

This retrospective study was done on records of 220 consecutive nephrectomies performed at a high-volume tertiary care urology center in a metropolitan city from June 2018 to June 2020. The series was analyzed according to the indications for nephrectomy, age group, clinical presentations, intraoperative and post-operative complications, and comorbidities. Ethical approval was obtained from the Institutional Ethics Committee. Waiver of consent was sought and no identifier information was used during data analysis. Data were entered in Microsoft Excel 2016 and analyzed using SPSS version 23.0.

\section{RESULTS}

This study conducted on records of 220 consecutive nephrectomies from June 2018 to June 2020 yielded the following results:

\section{Baseline characteristics}

Out of 220 nephrectomies, 149 (68\%) were performed for benign conditions, $37(17 \%)$ for malignant etiology, and $32(15 \%)$ cases were donor nephrectomy. In the benign group, the most common etiology was renal stones (98 patients, $44.54 \%$ ), followed by pelvic ureteric junction obstruction (PUJO) (28 patients, $12.72 \%$ ), renal tuberculosis (15 patients, $6.81 \%$ ), pyonephrosis (12 patients, $5.45 \%$ ), xanthogranulomatous pyelonephritis (11 patients, 5\%), and emphysematous pyelonephritis (four patients, $1.81 \%$ ). The most common age group affected by benign conditions was $30-45$ years (mean 37 years). Male and females were equally affected. The most common clinical presentations for benign conditions were flank pain (80\%), followed by lower urinary tract symptoms (LUTS) $(22 \%)$, dysuria $(25 \%)$, hematuria (15\%), and fever (7\%). Asymptomatic presentation with incidentally detected non-functioning kidney due to calculus disease and PUJO were 3\% and $2.5 \%$, respectively. Among 98 patients of calculus disease, 18 patients (19\%) suffered from hypertension alone, 12 patients $(13 \%)$ suffered from diabetes alone, 19 patients $(20 \%)$ had both diabetes with hypertension, and 17 patients $(18 \%)$ had chronic kidney disease (CKD); while 11 patients $(12 \%)$ had a history of tuberculosis. In the malignant group, the most common etiology was renal cell carcinoma (RCC) confined to the kidney (18 patients, $8.18 \%$ ), followed by RCC with inferior vena cava thrombosis (eight patients, $3.63 \%$ ), metastatic RCC (five patients, $2.27 \%$ ), angiomyolipoma (two patients, $0.9 \%$ ), and paraganglioma (one patient, $0.45 \%$ ). The most common age group for malignant conditions was 45-60 years (mean 52 years). Male (52\%) and female $(48 \%)$ were equally affected. The most common clinical presentations for malignant conditions were flank pain $(90 \%)$ followed by hematuria $(67 \%)$, LUTS $(10 \%)$, rarely fever (4\%), and vomiting (5\%). Among patients with malignancy, $27 \%$ were hypertensive, $25 \%$ were diabetic, $13 \%$ suffered from both diabetes mellitus (DM) and hypertension (HTN), 18\% suffered from CKD, and 5\% cases had a history of tuberculosis. Overall, 53 (24\%) patients presented with acute kidney injury (AKI). About $36 \%$ of the patients presented within 6 months of the onset of symptoms; whereas around $32 \%$ of patients had symptoms for more than 1 year before presentation. Other baseline characteristics are given in Table 1.

\section{Operative details}

A pre-operative intervention such as DJ stenting and percutaneous nephrostomy insertion was performed in $27(13 \%)$ and $19(9 \%)$ patients, respectively. A laparoscopic (lap) approach was used in $168(76 \%)$ of the patients out of which $24(10 \%)$ were converted to open procedures. The main reason for conversion to open procedures was hemorrhage (62\%), followed by dense adhesions leading to difficulty in dissections $(28 \%)$. Partial nephrectomy was performed in $8(3 \%)$ patients. Intra- and post-operative complications are given in Table 2.

\section{DISCUSSION}

In literature, there are very few audits about surgical nephrectomies. Kerbl et al., in 1994, analyzed laparoscopic nephrectomies; however, it is live-related donor nephrectomies alone. ${ }^{1}$ Beisland et al., 2000, ${ }^{2}$ Scott and Seizman in $1966,{ }^{3}$ and Schiff and Glazier, ${ }^{4} 1977$ are the few series available for reference. Naturally, indications for nephrectomy would vary from region to region, and a comparison would be futile. However, the number of nephrectomies performed for benign conditions in our study is much higher than that reported in the Western series. In our study, $68 \%$ of nephrectomies were done for benign etiology, $17 \%$ for malignant, and $15 \%$ were donor nephrectomy. This suggests that the people neglect 


\begin{tabular}{|c|c|c|}
\hline Baseline characteristics & Number & Percentage \\
\hline \multicolumn{3}{|l|}{ Age group (in years) } \\
\hline $11-20$ & 13 & 5.90 \\
\hline $21-30$ & 26 & 11.82 \\
\hline $31-40$ & 40 & 18.18 \\
\hline $41-50$ & 48 & 21.82 \\
\hline $51-60$ & 47 & 21.36 \\
\hline $61-70$ & 31 & 14.09 \\
\hline$>70$ & 15 & 6.82 \\
\hline \multicolumn{3}{|l|}{ Etiology } \\
\hline Benign & 149 & 67.73 \\
\hline Malignant & 37 & 16.82 \\
\hline Donor nephrectomy & 32 & 14.54 \\
\hline \multicolumn{3}{|l|}{ Symptoms at presentation } \\
\hline Flank pain & 156 & 70.90 \\
\hline Hematuria & 28 & 12.73 \\
\hline LUTS & 38 & 17.27 \\
\hline Dysuria & 46 & 20.90 \\
\hline Fever & 18 & 8.18 \\
\hline Nausea/vomiting & 18 & 8.18 \\
\hline Asymptomatic & 6 & 2.73 \\
\hline \multicolumn{3}{|c|}{$\begin{array}{l}\text { Duration of symptom onset to doctor } \\
\text { consultation }\end{array}$} \\
\hline$<6$ months & 79 & 35.90 \\
\hline 6 months -1 year & 69 & 31.36 \\
\hline 1 year-2 years & 51 & 23.18 \\
\hline$>2$ years & 21 & 9.54 \\
\hline \multicolumn{3}{|l|}{ Comorbidities } \\
\hline $\mathrm{DM}$ & 28 & 12.72 \\
\hline HTN & 42 & 19.09 \\
\hline Tuberculosis & 26 & 11.81 \\
\hline CKD & 39 & 17.72 \\
\hline $\mathrm{DM}+\mathrm{HTN}$ & 44 & 20 \\
\hline None & 40 & 18.18 \\
\hline
\end{tabular}

symptoms, take alternate therapies, and present late with non-functioning kidneys. The mean age of the patients with benign indications was 37 years and with malignant indications was 52 years. No sex predilection was seen in benign and malignant conditions. Similarly, in a study by Rafique in Pakistan, $76 \%$ nephrectomies were done for benign etiology and $23 \%$ for malignant etiology. The mean age of the patients with benign indications was 32 years and with malignant indications was 53 years. The benign conditions were more in females and malignant conditions were more in males. ${ }^{5}$ However, in the Western series, a study conducted by Beisland et al., $67 \%$ nephrectomies were done for malignant indications and $23 \%$ for benign conditions. The mean age of the patients with benign indications was 51 years and with malignant indications was 65 years. ${ }^{2}$ In our study, $45 \%$ of the total nephrectomies were done for calculus disease and its complications. Therefore, it is necessary to see whether this can be prevented in the future. Emphasis should be placed on public awareness with the help of the community medicine department.

\begin{tabular}{lcc}
$\begin{array}{l}\text { Table 2: Operative details and complications in } \\
\text { patients }(\mathbf{n = 2 2 0})\end{array}$ & Number & Percentage \\
\hline Operative details & 129 & 58.64 \\
\hline Surgical approach & 38 & 17.27 \\
$\quad$ Lap simple nephrectomy & 19 & 8.64 \\
Open simple nephrectomy & & \\
Lap converted to open simple & 5 & 2.27 \\
nephrectomy & & \\
Lap converted to open radical & 12 & 5.45 \\
nephrectomy & 9 & 4.09 \\
Open radical nephrectomy & 8 & 3.64 \\
Lap radical nephrectomy & & \\
Lap partial nephrectomy & 28 & 12.73 \\
Intra-operative complications & 12 & 5.45 \\
Hemorrhage > 300 ml & 8 & 3.64 \\
Major vessel injury & 1 & 0.45 \\
Pleural injury & 1 & 0.45 \\
Duodenal injury & & \\
Ascending colon tear & 14 & 6.36 \\
Post-operative complications & 8 & 3.64 \\
SSI & 8 & 3.64 \\
Pulmonary complications & 5 & 2.27 \\
Fever & 1 & 0.45 \\
Urinoma & 1 & 0.45 \\
PCS leak & 1 & 0.45 \\
Abscess & & \\
Intra-abdominal collection & & \\
\hline SSI: Surgical site infections, PCS: Pelvi-collecting system & & \\
& &
\end{tabular}

About $62 \%$ of our patients underwent laparoscopic nephrectomy and 16\% underwent open nephrectomy. Conversion to open nephrectomy was done in $12 \%$. The rate of complication was $12.7 \%$. Studies done in the past show majority of the nephrectomies were open. With the advancement of technology, laparoscopic nephrectomy is possible now with fewer complications, lesser reoperation rates, and fewer mortalities. ${ }^{2,5}$

About $24 \%$ of the patients who underwent nephrectomy presented with AKI, which has a high mortality rate. Others had symptoms related to stones whereas only $3 \%$ were asymptomatic with incidental detection.

Almost $35 \%$ of the patients presented within 6 months of the onset of symptoms. Thus, it strongly suggests a need for a screening method for the high-risk cases, (i.e., patients with comorbidities such as DM, HTN, history of tuberculosis, and $\mathrm{CKD}$ ) preferably during their visit to endocrine or nephrology OPDs, facilitating early recognition of calculus disease and prompt intervention. Another $32 \%$ of patients presented after 1 year from symptom onset. The primary reason for late referral was a lack of awareness and seriousness about calculus disease and its implications, self-medication fueled by misinformation that it will cause stone dissolution, and visits to alternate medicine practitioners leading to inappropriate treatment. Thus, awareness campaigns involving local electronic and print media are the need of the hour so that appropriate 
knowledge is acquired and precious time is not wasted in seeking expert opinion.

\section{Limitations of the study}

1) Complete Follow up data weren't available due to ongoing covid pandemic situation. 2) It was single center study. (On the basis of encouraging results of this study, A pan-India multi-centre study is needed to better extapolate the findings.

\section{CONCLUSION}

There is a drastic difference in nephrectomy indications between Western countries and India. In our study, about $68 \%$ of nephrectomies were performed for calculus disease and its complications. Nephrectomy for calculus disease is potentially preventable. Screening procedures particularly for the high-risk group of patients, creating public awareness and educating them through involvement of local electronic and print media as well as social media campaigns are the need of the hour. Involvement of the Department of Community Medicine for the same and encouraging screening of the high-risk cases in endocrinology and nephrology OPDs will prove to be highly beneficial.

\section{ACKNOWLEDGMENT}

We would like to acknowledge all my patients without whom the study wouldn't have been possible, my colleagues for their constant support, and my teachers for their guidance.

\section{REFERENCES}

1. Kerbl K, Clayman R, McDougall E, Gill I, Wilson B, Chandhoke P, et al. Transperitoneal nephrectomy for benign disease of the kidney: A comparison of laparoscopic and open surgical techniques. Urology. 1994;43(5):607-613.

https://doi.org/10.1016/0090-4295(94)90171-6

2. Beisland C, Medby PC, Sander S and Beisland HO. Nephrectomyindications, complications and postoperative mortality in 646 consecutive patients. Eur Urol. 2000;37(1):58-64.

https://doi.org/10.1159/000020101

3. Scott RF and Selzman,HM. Complications of nephrectomy: Review of 450 patients and a description of a modification of the transperitoneal approach. J Urol. 1966;95(3):307-312. https://doi.org/10.1016/s0022-5347(17)63452-9

4. Schiff $M$ and Glazier, WB. Nephrectomy: Indications and complications in 347 patients. J Urol. 1977;118(6):930-931. https://doi.org/10.1016/s0022-5347(17)58253-1

5. Rafique M. Nephrectomy: Indications, complications and mortality in 154 consecutive patients. J Pak Med Assoc. 2007;57(6):308-311.

\footnotetext{
Authors Contribution:

AV- Concept and design of the study, prepared first draft of manuscript; KS, TR- Interpreted the results; reviewed the literature and manuscript preparation;

SB, BP, SP- Concept, coordination, statistical analysis and interpretation, and revision of the manuscript

Work attributed to:

Department of Urology, Seth GS Medical College and KEM Hospital, Mumbai - 400 012, Maharashtra, India

Orcid ID:

Dr. Kamlesh Singh - (D) https://orcid.org/0000-0002-7156-8299

Dr. Tarun Rochlani - (i) https://orcid.org/0000-0002-5501-1909

Dr. Sachin Bhujbal - (1) https://orcid.org/0000-0001-6112-6916

Dr. Bhushan Patil - id https://orcid.org/0000-0001-8679-7687

Dr. Sujata Patwardhan - (1) https://orcid.org/0000-0003-3676-1015

Source of Funding: None, Conflicts of Interest: None.
} 